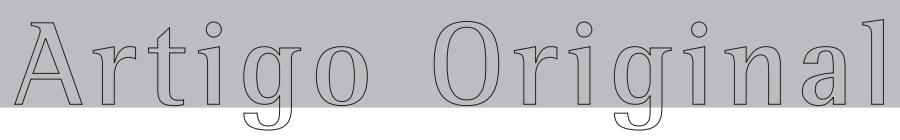

\title{
Teste de caminhada de seis minutos: estudo do efeito do aprendizado em portadores de doença pulmonar obstrutiva crônica*
}

\author{
Six Minutes Walk Test: Study of the Effect of Learning in Chronic Obstructive \\ Pulmonary Disease Patients.
}

SÉRGiO LEITE RODRIGUES, HÉLDER FONSECA E MENDES, CARLOS ALBERTO DE ASSIS VIEGAS(TE SBPT)

\begin{abstract}
Introdução: Testes de caminhada de seis minutos vêm sendo utilizados de forma crescente para avaliar a efetividade de diferentes opções terapêuticas clínicas e cirúrgicas em pneumopatias. Entretanto, a falta de padronização para a sua realização pode influenciar as aferições, prejudicando a qualidade da avaliação. Nesse sentido, formulamos a hipótese de que os pacientes têm melhor performance com a realização do teste de caminhada de seis minutos após aprendizado.
\end{abstract}

Objetivo: Determinar o possível efeito do aprendizado na distância percorrida durante o teste de caminhada de seis minutos em portadores de doença pulmonar obstrutiva crônica.

Método: Foram analisados, retrospectivamente, 35 prontuários de pacientes portadores de doença pulmonar obstrutiva crônica, encaminhados ao Programa de Reabilitação Pulmonar do Hospital Universitário de Brasília, e que tivessem realizado, em dias alternados, dois testes de caminhada de seis minutos, espirometria e gasometria arterial. 0 diagnóstico clínico e funcional de doença foi baseado na história de exposição a fator de risco, produção de secreção, dispnéia e prova espirométrica alterada, após o uso de broncodilatador.

Resultados: Observamos que as distâncias percorridas no segundo teste de caminhada de seis minutos $(515 \pm 82$ metros) foram maiores que as distâncias percorridas no primeiro ( $480 \pm 85$ metros), com valores estatisticamente significativos $(p<0,05)$. Entretanto, as variáveis esforço muscular, percepção da dispnéia (escala de Borg), saturação periférica da hemoglobina pelo oxigênio, freqüência respiratória e freqüência cardíaca não apresentaram diferença significativa entre os dois testes $(p<0,05)$.

Conclusão: 0 presente estudo sugere a necessidade de padronização do teste de caminhada de seis minutos, com a realização de pelo menos dois testes para se avaliar a capacidade funcional de pacientes portadores de doença pulmonar obstrutiva crônica.

J Bras Pneumol 2004; 30(2) 121-125
Background: The six minutes walk test has been increasingly utilized to assess the effectiveness of different clinical and surgical treatment options in pulmonary diseases. However lack of standardization for their performance may influence measurements and jeopardize assessment of the functional capacity of patients with cardiopulmonary disease.

Objective: To determine the possible effects of learning on the distance covered during the six minute walk test for bearers of chronic obstructive pulmonary disease.

Method: A retrospective analysis of 35 medical records of COPD patients referred to the Pulmonary Rehabilitation Program of the University Hospital of Brasilia was carried out. On alternate days these patients had performed two six minutes walk tests, spirometry and arterial blood gas analysis. Clinical and functional diagnosis was based upon the history of exposure to risk factors, mucus production, dyspnea and spirometric dysfunction after use of bronchodilators. The Student test was used for the comparison of results that were different.

Results: The distances covered in the second six-minute walk test ( $515 \pm 82$ meters) were greater than those covered in the first six-minute walk test ( $480 \pm 85$ metros), with statistically significant differences $(p<0.05)$. However measurements of the muscular effort and perception of dyspnea (Borg scale), peripheral blood oxygen saturation $\left(\mathrm{SpO}_{2}\right)$, respiratory and heart rates did not disclose any statistically significant differences between the two tests $(\mathrm{p}<0.05)$.

Conclusion: The present study suggests that in order to standardize the six minutes walk test, the procedure should be performed at least twice to better assess the functional capacity of patients, bearers of chronic obstructive pulmonary disease.

Key words: Walking. Effectiveness. Lung diseases, obstrutive. 


\section{INTRODUÇÃO}

A corrida de doze minutos foi inicialmente padronizada por Cooper, para avaliar a capacidade física em indivíduos saudáveis ${ }^{1}$, e modificada para caminhada de doze minutos para avaliar a capacidade física de pacientes com bronquite crônica ${ }^{2}$. Posteriormente, Butland et al. exploraram com sucesso a utilização dos testes de caminhada com duração de dois, seis e doze minutos na aferição da capacidade física de portadores de doença pulmonar obstrutiva crônica (DPOC) ${ }^{3}$. Desde então, os testes de caminhada vêm sendo utilizados de forma crescente, para avaliar a efetividade de diferentes modalidades de tratamento nas áreas clínica e cirúrgica ${ }^{4}$.

No que se refere à DPOC, sabe-se que esta condição pode levar à incapacidade física, desencadeando limitações físicas e sociais que acarretam deterioração da qualidade de vida de seus portadores ${ }^{4}$. Na avaliação da capacidade física, os testes de caminhada de seis minutos (TC6) também avaliam a capacidade funcional ou a habilidade de empreender atividades na vida diária. Essa aferição tem se mostrado importante na avaliação dinâmica e no manejo clínico de individuos com doenças cardiopulmonares crônicas graves, que não apresentam condições clínicas para a realização de provas físicas com esforço máximo ${ }^{4,5}$.

No Brasil, o I Consenso Brasileiro de DPOC e as recomendações da Sociedade Brasileira de Pneumologia e Tisiologia (SBPT) para os testes de função pulmonar não contemplaram a realização ou a padronização do TC6 para a avaliação funcional de pacientes portadores de DPOC $^{6,7}$. Recentemente, a American Thoracic Society (ATS) sugeriu que a realização de um TC6 como treino pode melhorar a coordenação motora e diminuir a ansiedade dos pacientes em testes subseqüentes. Este procedimento pode conferir maior confiabilidade nos resultados dos testes, tendo em vista a redução da influência dos fatores neuromusculares e psicológicos inerentes à população de portadores de $\mathrm{DPOC}^{8-10}$.

Pela falta de consenso e pela escassez de estudos na literatura nacional, o objetivo deste trabalho foi determinar o possível efeito do aprendizado na distância percorrida durante o TC6 em portadores de DPOC atendidos em nosso hospital.
Siglas e abreviaturas utilizadas neste trabalho:

CPT - Capacidade pulmonar total

CVF- Capacidade vital forçada

DPOC - Doença pulmonar obstrutiva crônica

IMC - Índice de massa corpórea

$\mathrm{pH}$ - 0 logaritmo negativo, para a base 10 , da concentração de íons livres de hidrogênio em uma solução

$\mathrm{PaCO}_{2}$ - Pressão parcial de $\mathrm{CO}_{2}$ no sangue arterial

$\mathrm{PaO}_{2}$ - Pressão parcial de $\mathrm{O}_{2}$ no sangue arterial

$\mathrm{SpO}_{2}$ - Saturação periférica da hemoglobina pelo oxigênio

TC6 - Teste de caminhada de seis minutos

$1^{\circ}$ TC6 - Primeiro teste de caminhada de seis minutos

$2^{\circ}$ TC6 - Segundo teste de caminhada de seis minutos

VEF - Volume expiratório forçado no primeiro segundo

$\mathrm{VEF}_{1} / \mathrm{CVF} \%$ - Razão entre volume expiratório forçado no primeiro segundo e a capacidade vital forçada, expresso como percentagem

\section{MÉTODO}

Foi feito um estudo retrospectivo, analisandose os prontuários de pacientes submetidos ao Programa de Reabilitação Pulmonar do Hospital Universitário de Brasília, no período de outubro de 2002 a maio de 2003.

Dos prontuários revisados, foram analisadas as variáveis espirométricas, gasométricas, o primeiro $\left(1^{\circ} \mathrm{TC} 6\right)$ e o segundo ( $\left.2^{\circ} \mathrm{TC} 6\right)$ testes de caminhada de seis minutos.

$01^{\circ} \mathrm{TC} 6$ e o $2^{\circ} \mathrm{TC} 6$ foram realizados segundo as recomendações da ATS $^{9}$. Os pacientes realizaram os testes em dias alternados. 0 primeiro teve o propósito de avaliar a distância percorrida sem o efeito do aprendizado, e o segundo aferir os valores, com a possibilidade de efeito do aprendizado e adaptação ao teste. Os parâmetros aferidos antes e após os testes foram a freqüência respiratória, freqüência cardíaca, saturação periférica da hemoglobina pelo oxigênio $\left(\mathrm{SpO}_{2}\right)$, percepção do esforço muscular e da dispnéia pela escala modificada de Borg ${ }^{11}$ e a distância total percorrida. As equações propostas por Enright e Sherrill ${ }^{12}$ foram utilizadas para determinar os valores de normalidade da distância percorrida.

A SpO $\mathrm{S}_{2}$ no sangue arterial foi aferida no início e no final de ambos os testes ( $1^{\circ} \mathrm{TC} 6$ e $2^{\circ}$ TC 6 ), com o sensor no dedo indicador do membro superior dominante e o oxímetro fixado na cintura do paciente. 0 aparelho utilizado para aferição da $\mathrm{SpO}_{2}$ foi o oxímetro de pulso 920M, Healthdyne technologies ${ }^{\circledR}$, Marietta-GA, USA. 
A espirometria foi realizada em três manobras de expiração forçada com os indivíduos na posição sentada, e os critérios de aceitabilidade e reprodutibilidade foram os recomendados pela $\mathrm{ATS}^{13}$. As variáveis estudadas foram a capacidade vital forçada (CVF), o volume expiratório forçado no primeiro segundo $\left(\mathrm{VEF}_{1}\right)$ e a relação $\mathrm{VEF}_{1} /$ CVF\%. Foram medidos os valores absolutos e calculados os percentuais relativos aos previstos para sexo, idade e altura, seguindo a tabela descrita por Knudson et al. ${ }^{14}$. 0 espirômetro utilizado foi o Vmax - 22 series spirometer SensorMedics Yorba Linda, California, USA.

A gasometria arterial foi realizada estando o indivíduo em repouso e a amostra de sangue colhida através da punção da artéria radial do membro não dominante, após anestesia com xylocaína. Foram colhidos $2 \mathrm{ml}$ de sangue, imediatamente processados em um analisador de gás computadorizado e automatizado, para medir os valores da $\mathrm{PaO}_{2}$ e $\mathrm{PaCO}_{2}$ expressos em mmHg e da $\mathrm{SaO}_{2}$ em porcetagem de saturação. 0 aparelho utilizado para analisar as amostras foi o Ciba Corning 278 Gas System ${ }^{\circledR}$, Ciba Corning, Diagnostics Corp; Medifield, USA, calibrado automaticamente todas as manhãs, antes do início dos exames.

Foram incluidos todos os prontuários de pacientes portadores de DPOC, encaminhados ao Programa de Reabilitação Pulmonar do Hospital Universitário de Brasília, que tenham realizado e documentado o $1^{\circ} \mathrm{TC} 6$, o $2^{\circ} \mathrm{TC} 6$, a espirometria e a gasometria arterial. 0 diagnóstico clínico e funcional de DPOC foi baseado na história de exposição ao fator de risco, produção de secreção, dispnéia e prova espirométrica alterada após o uso de broncodilatador ${ }^{15}$.

Foram excluídos aqueles pacientes que por qualquer motivo não tenham completado o $1^{\circ} \mathrm{TC} 6$ e/ou o $2^{\circ}$ TC6, assim como aqueles que não realizaram gasometria e/ou espirometria.

Os dados obtidos são apresentados em seus valores médios e desvios padrão e a comparação entre os resultados obtidos nos dois testes foi feita pelo teste $\mathrm{t}$ de Student. Foram consideradas estatisticamente significativas as diferenças com $p<0,05$.

\section{RESULTADOS}

Foram selecionados 38 pacientes, contudo, 3 foram excluidos devido à falta de exames nos prontuários. Portanto, foram estudados os prontuários de 35 pacientes, 28 homens e 7 mulheres. A média de idade foi de $65 \pm 8$ anos, com a mínima de 46 anos e a máxima de 81 anos. 0 índice de massa corpórea (IMC) médio foi de 24 $\pm 4 \mathrm{Kg} / \mathrm{m}^{2}$, com o valor mínimo de $15 \mathrm{Kg} / \mathrm{m}^{2}$ e o valor máximo de $31 \mathrm{Kg} / \mathrm{m}^{2}$. $0 \mathrm{VEF}_{1}$ variou de um valor mínimo de 38\% a um valor máximo de $86 \%$ em relação aos valores previstos, com valor médio de $62 \% \pm 24 \%$. Em relação à CVF, observamos um valor mínimo de 46\% e um valor máximo de 120\% em relação aos valores previstos, com valor médio de $95 \pm 25 \%$. 0 indice $\mathrm{VEF}_{1} / \mathrm{CVF} \%$ apresentou um valor mínimo de 24\%, um valor máximo de $66 \%$ e médio de $52 \pm 14 \%$. Observamos, na gasometria arterial, o valor médio do $\mathrm{pH}$ de 7,4 \pm 0 . Com relação à $\mathrm{PaO}_{2}$, encontramos uma média de $74 \pm 9 \mathrm{mmHg}$ com um valor mínimo de $65 \mathrm{mmHg}$ e um valor máximo de $83 \mathrm{mmHg}$. $\mathrm{A} \mathrm{PaCO}_{2}$ média foi de $37 \pm$ $11 \mathrm{~mm} \mathrm{Hg}$ com valor mínimo e máximo de $26 \mathrm{~mm} \mathrm{Hg}$ e $48 \mathrm{mmHg}$, respectivamente (Tabela 1).

Em relação à aferição das variáveis de esforço muscular, percepção da dispnéia (escala de Borg), $\mathrm{SpO}_{2}$, freqüência respiratória e freqüência cardíaca, não houve diferença estatisticamente significativa entre os valores obtidos no $1^{\circ} \mathrm{TC} 6$ e no $2^{\circ} \mathrm{TC} 6$. A distância percorrida durante o $1^{\circ}$ TC6 foi de 480 \pm 85 metros com mínima e máxima percorridas de 277 e 616 metros, respectivamente. No $2^{\circ}$ TC6, a distância média foi de $515 \pm 82$ metros, com mínima de 280 metros e máxima de 640 metros. Quando comparamos a diferença entre as distâncias percorridas nos dois testes, encontramos um valor estatisticamente significativo (Tabela 2).

\section{DISCUSSÃO}

A reabilitação pulmonar tem-se mostrado eficaz na melhoria da capacidade funcional e na força muscular de portadores de DPOC, mesmo não havendo ganhos nos testes de função pulmonar ${ }^{16,17}$. O TC6 é freqüentemente utilizado para avaliar a capacidade física e evolução do paciente portador de DPOC ${ }^{9}$. Solway et al. afirmam que o TC6 é utilizado por $87 \%$ dos programas de reabilitação pulmonar nos EUA e Canadá. A simplicidade, o baixo custo e a boa correlação com a capacidade física submáxima são os principais fatores que incentivam sua aplicaçã $0^{5}$. Segundo Rodrigues et al. ${ }^{18}$, o TC 6 correlaciona-se de forma positiva e significativa com indicadores de 
prognóstico da DPOC, como a $\mathrm{PaO}_{2}$ e o $\mathrm{VEF}_{1}$.

Em estudos sobre o TC6, a influência do aprendizado é citada como fator de interferência nos resultados e na reprodutibilidade do teste ${ }^{8-10}$. Contudo, não encontramos consenso da utilização do TC6 de aprendizado na aferição da capacidade funcional do paciente portador de DPOC. Em recente publicação, Moreira et al. ${ }^{19}$, ao avaliarem os TC6 realizados em um programa de reabilitação pulmonar, citaram a importância da padronização

TABELA 1

Características antropométricas e funcionais da população estudada

\begin{tabular}{cc}
\hline Sexo & 28 homens 7 mulheres \\
ldade (anos) & $65 \pm 08$ \\
IMC $(\mathrm{Kg} / \mathrm{m} 2)$ & $24 \pm 04$ \\
$\mathrm{CVF}(\%)$ & $95 \pm 25$ \\
$\mathrm{VEF} 1(\%)$ & $62 \pm 24$ \\
$\mathrm{VEF} 1 / \mathrm{CVF} \%$ & $52 \pm 14$ \\
$\mathrm{pH}$ & $7,4 \pm 00$ \\
$\mathrm{PaO} 2(\mathrm{mmHg})$ & $74 \pm 09$ \\
$\mathrm{PaCO} 2(\mathrm{mmHg})$ & $37 \pm 11$ \\
\hline
\end{tabular}

IMC: índice de massa corpórea; CVF: capacidade vital forçada; $\mathrm{VEF}_{1}$ : volume expiratório forçado no primeiro segundo; $\mathrm{PaO}_{2}$ : pressão parcial de oxigênio no sangue arterial; $\mathrm{PaCO}$ : pressão parcial de gás carbônico no sangue arterial.

TABELA 2

Resultados do $1^{\circ} \mathrm{TC} 6$ e $2^{\circ} \mathrm{TC} 6, \mathrm{SpO}_{2}$ inicial e final, e avaliação da dispnéia

\begin{tabular}{lcc}
\hline & $1^{\circ} \mathrm{TC} 6$ & $2^{\circ} \mathrm{TC} 6$ \\
\hline Distância (metros) & $480 \pm 85$ & $515 \pm 82^{*}$ \\
FC inicial (bpm) & $81 \pm 10$ & $77 \pm 10$ \\
FC final (bpm) & $111 \pm 13$ & $119 \pm 18$ \\
FR inicial (ipm) & $16 \pm 03$ & $16 \pm 04$ \\
FR final (ipm) & $24 \pm 03$ & $24 \pm 04$ \\
Sp02 inicial & $94 \pm 03$ & $94 \pm 03$ \\
Sp02 final & $91 \pm 05$ & $89 \pm 4,8$ \\
Borg muscular & $04 \pm 03$ & $04 \pm 03$ \\
Borg respiratório & $04 \pm 03$ & $04 \pm 02$ \\
\hline
\end{tabular}

$1^{\circ}$ TC6: primeiro teste de caminhada de seis minutos; $2^{\circ} \mathrm{TC} 6$ : segundo teste de caminhada de seis minutos; FC: freqüência cardíaca; FR: freqüência respiratória; Sp02: saturação periférica da hemoglobina pelo oxigênio.

* $p<0,05$ em relação ao primeiro teste do TC6. Os autores sugerem que os principais fatores do aumento de rendimento físico são o estímulo verbal e a forma de acompanhamento do paciente durante o TC6. Em nosso trabalho, entretanto, observamos diferença importante $(\mathrm{p}<0,05)$ entre 0 $1^{\circ} \mathrm{TC} 6$ e $2^{\circ} \mathrm{TC} 6$, mesmo acompanhando os pacientes e utilizando estímulo verbal idêntico. Para Knox et al., aumentos de 33\% nas distâncias percorridas são encontrados em caminhadas repetidas, utilizando o mesmo estímulo verbal no TC6. Nesse estudo, os autores realizaram o TC6 doze vezes durante três dias consecutivos e observaram ganho de 16\% nas distâncias percorridas até o terceiro teste $^{4}$. Em nosso estudo, utilizando apenas dois testes, observamos um aumento médio de $8 \%$, com diferença estatisticamente significativa em relação à distância percorrida. Isto sugere uma maior adaptação do paciente em relação aos aspectos práticos do teste, tais como o controle da ansiedade, o reconhecimento dos limites da prova e a adaptação neuromuscular em relação à atividade a ser realizada. Em sua metanálise, Lacasse et al. ${ }^{20}$ observaram que os ganhos clínicos significativos do paciente submetido à reabilitação pulmonar ocorrem com aumentos aproximados de 50 metros no TC6, com variações entre 27,8 e 92,8 metros. No presente trabalho, observamos valores que estão entre os citados na literatura ${ }^{20,21}$, com aumento de 35 metros na distância média após o $1^{\circ} \mathrm{TC} 6$. Acreditamos que a diferença estatística encontrada entre o $1^{\circ} \mathrm{TC} 6$ e o $2^{\circ} \mathrm{TC} 6$, em nosso estudo, justifica a realização do $1^{\circ}$ TC6. Em caso contrário, estaríamos superestimando os resultados das intervenções terapêuticas em relação à capacidade funcional dos pacientes, principalmente daqueles com doença mais avançada.

Para Noseda et al. ${ }^{21}$, em estudo sobre a reprodutibilidade dos testes físicos para o portador de DPOC, o efeito aprendizado em curtos períodos é freqüentemente relevante, com estimulação verbal e repetição do TC6. Os autores, sem o uso da estimulação verbal, observaram um aumento médio de 24,5 metros em três repetições do teste em três meses consecutivos. Para Redelmeier et al. ${ }^{10}$, a realização do TC6 em dias consecutivos é suficiente para o controle de possíveis efeitos de aprendizado. Esses autores sugerem que após a realização de dois testes, chega-se a um platô, e não há diferença estatisticamente significativa entre os testes realizados posteriormente. 
Em relação à distância percorrida, nossos resultados, seja no $1^{\circ} \mathrm{TC} 6$ ou no $2^{\circ} \mathrm{TC} 6$, concordam com os valores de referência para indivíduos saudáveis sugeridos por Enright et al. ${ }^{12}$. Entretanto, utilizamos o encorajamento verbal a cada minuto em ambos os testes, o que pode explicar o melhor rendimento físico dos pneumopatas do nosso estudo, em relação aos indivídios estudados por esses autores.

No que se refere às variáveis freqüência respiratória, freqüência cardíaca e percepção do esforço muscular e respiratório (escala de Borg), não observamos diferenças significativas $(p>0,05)$ entre o $1^{\circ} \mathrm{TC} 6$ e o $2^{\circ} \mathrm{TC} 6$. Isto sugere que o grau de esforço desenvolvido pelos pacientes foi semelhante entre os testes, e corrobora a hipótese de que o fator determinante para o aumento significativo no $2^{\circ} \mathrm{TC} 6$ foi o aprendizado.

A falta de padronização do TC6, que pode influenciar os resultados do teste e impossibilitar a comparação entre resultados obtidos em pesquisas distintas ${ }^{2}$, é uma questão que está inserida no contexto nacional ${ }^{16-19}$.

Concluímos que, na população estudada, o $1^{\circ}$ TC6 mostrou uma diferença estatisticamente significativa em relação ao $2^{\circ} \mathrm{TC} 6$. Acreditamos que a realização de mais de um teste de caminhada de seis minutos deve ser considerada durante o processo de avaliação funcional do pneumopata crônico. Este procedimento poderá fornecer maior qualidade e segurança na aferição da capacidade física, bem como na avaliação dos resultados terapêuticos de portadores de DPOC.

\section{REFERÊNCIAS}

1. Cooper KH. A means of assessing maximal oxygen intake. JAMA 1968;203:201-4.

2. McGavin CR, Gupta SP, McHardy GJR. Twelve-minute walking test for assessing disability in chronic bronchitis. Br Med J 1976;1:822-3.

3. Butland RJ, Pang J, Cross ER, Woodcock AA, Geddes DM. Two-six, and 12-minute walking test in respiratory disease. BMJ 1982;284:1607-8.

4. Knox AJ, Morrison JFJ, Muers MF. Reproducibility of walking test results in chronic obstructive airways disease. Thorax 1988;43:388-92.
5. Solway S, Brooks D, Lacasse Y, Thomas S. A qualitative systematic overview of the measurement properties 0 functional walk tests used in the cardiorespiratory domain. Chest 2001;119:256-70.

6. I Consenso Brasileiro de Doença Pulmonar Obstrutiva Obstrutiva Crônica (DPOC). J Pneumol 2000;26:S34-S52.

7. Diretrizes para Testes de Função Pulmonar. J Pneumol 2002;28:S166-S206.

8. Elpern EH, Stevens D, Kesten S. Variability in performance of timed walk tests in pulmonary rehabilitation programs. Chest 2000;118:98-105.

9. American Thoracic Society. ATS statement: guidelines for six-minute walk test. Am J Respir Crit Care Med 2002;166:111-7.

10. Redelmier DA, Bayoumi AM, Goldstein RS, Guyatt GH. Interpreting small differences in funtional status: the six minute walk test in chronic lung disease patients. Am J Respir Crit Care Med 1997;155:1278-82.

11.Wilson RC, Jones PW. A comparison of the visual analogue scale and modified Borg scale for measurement of dyspnea during exercise. Clin Sci 1989;76:277-82.

12. Enright PL, Sherrill DL. Reference equations for the sixminute walk in healthy adults. Am J Respir Crit Care Med 1998;158:1384-7.

13. American Thoracic Society. Lung function testing: Selection of reference values and interpretative strategies. Am Rev Respir Dis 1991;144:1202-18.

14. Knudson RJ, Lebowitz MD, Holberg CJ, Burrows B. Changes in the normal expiration flow-volume curve with growth and aging. Am Rev Respir Dis 1983;127:725-34.

15. NHLBl/WHO Global Initiative for Chronic Obstrutive Lung Disease (GOLD) Workshop Summary. Global strategy for the diagnosis, management, and prevention of chronic obstructive pulmonary disease. Am J Respir Crit Care Med 2001;163:1256-76.

16. Rodrigues SL, Viegas CAA, Lima T. Efetividade da reabilitação como tratamento coadjuvante da doença pulmonar obstrutiva crônica. J Pneumol 2002;28:65-70.

17. Neder JA, Nery LE, Filha SPC, Ferreira IM, Jardim JR. Reabilitação pulmonar: fatores relacionados ao ganho aeróbio de pacientes com DPOC. J Pneumol 1997;23:115-23.

18. Rodrigues SL, Viegas CAA. Estudo de correlação entre provas funcionais respiratórias e o teste de caminhada de seis minutos em pacientes portadores de doença pulmonar obstrutiva crônica. J Pneumol 2002;28:324-8.

19. Moreira AMC, Moraes MR, Tannus R. Teste da caminhada de seis minutos em pacientes com DPOC durante programa de reabilitação. J Pneumol 2001;27:295-300.

20. Lacasse Y, Wong E, Guyatt GH, King D, Cook DJ, Goldstein R. Meta-analise of respiratory rehabilitation in chronic obstructive pulmonary disease. Lancet 1996;348:1115-9.

21. Noseda A, Carpiaux JP, Prigogine T, Schmerber J. Lung function, maximum and submaximum exercise testing in COPD patients: reproducibility over a long interval. Lung 1989;167:247-57. 